
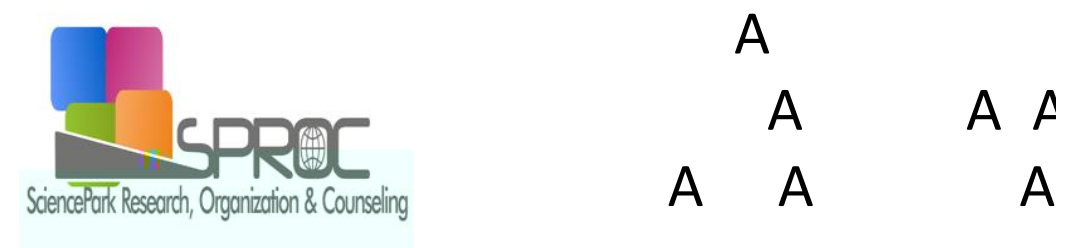

W A A A A A A

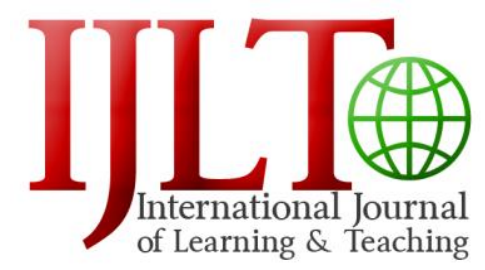

http://sproc.org/ojs/index.php/ijlt

\title{
What does giftedness mean according to teachers?
}

Deniz Ozcan ",A A A Furkan Kaya,A A A A

A

Suggested Citation:

A A A AGA A A A Learning and Teaching \&s $A \quad A$

$\begin{array}{cccccccc}\mathrm{A} & \mathrm{A} & \mathrm{A} & \mathrm{A} & \mathbb{N} & \mathrm{A} & \mathrm{A} & \mathrm{A} \\ \mathrm{A} & \mathrm{A} & \mathrm{A} & \mathrm{A} & \mathbb{N} & \mathrm{A} & \mathrm{A} & \mathrm{A}\end{array}$

A A

A A

Anternational Journal of A
AG C A
A $A$ A
A A A
A A A A

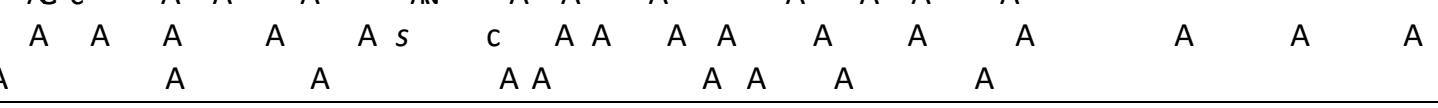

\section{Abstract}

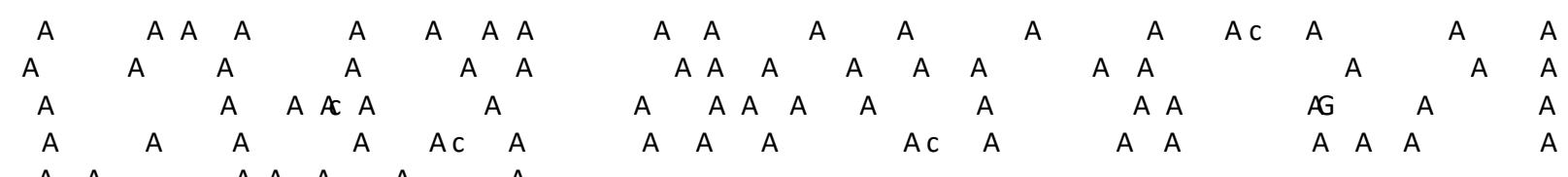
$A$
A
A A A
A
A
A A

A A A A

A

A

A

A

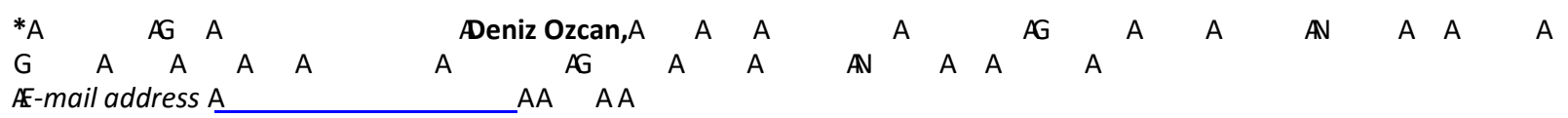




\section{Introduction}

$\begin{array}{llllllllllllllllll}A & A & A & A & A & A & A & A & A & A & A & A & A & A\end{array}$ $\begin{array}{lllllllllllll}A & A & A & A & A & A & A & A & A & A & A & A & A\end{array}$

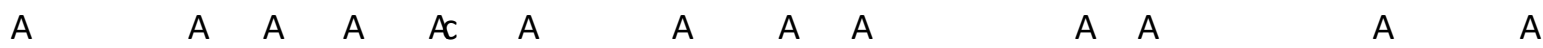
A A A A A A A A A A A A $\quad$ A $\quad$ A A $A$

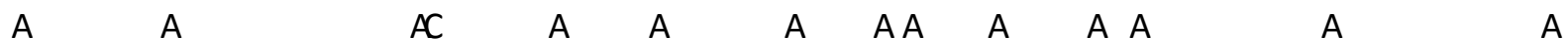
$\begin{array}{llllllllllllll}A & A & A & A & A & A & A & A & A & A & A & A & A & A\end{array}$ c A

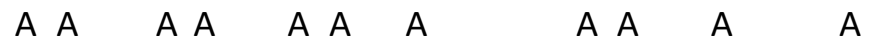

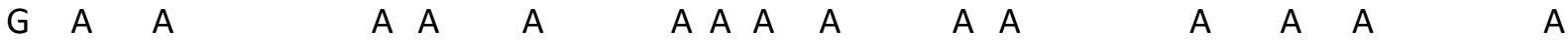

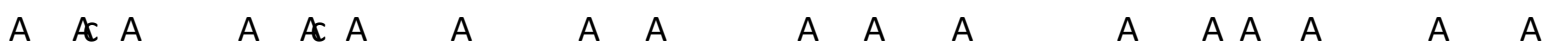

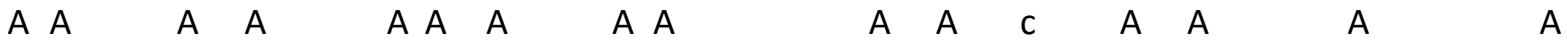
A $A$ A $A A$ A $\quad A \quad A \quad A \quad A \quad A \quad A$ AA AAA $A$ $\begin{array}{lllll}A & A & A & A & A\end{array}$

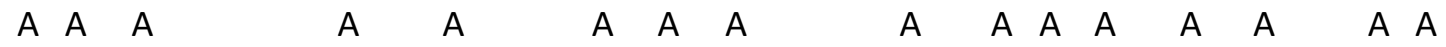
$\begin{array}{llllllllllllll}A & A & A & A & A & A & A & C & A & A & A & A & A A A\end{array}$ $\begin{array}{llllllllllllll}A & A & C & A & A & A & A & A & A & A & A & A & A & A\end{array}$

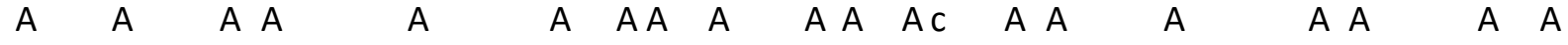

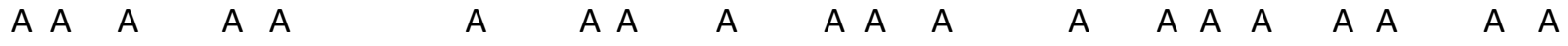

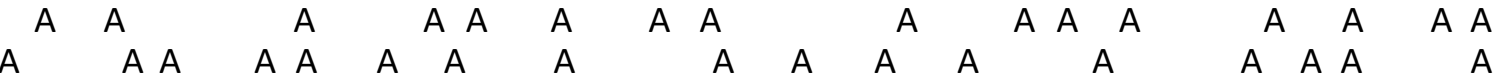
A A

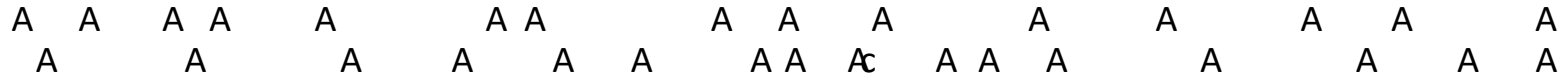

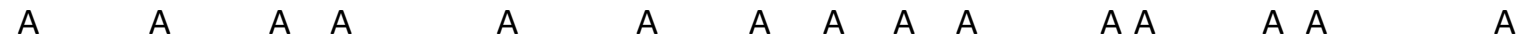

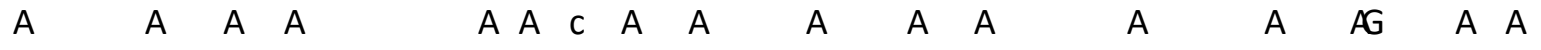
$N \quad A \quad A \quad A \quad A \quad A A A \quad A A$ A A A A A A A CA $A \quad A \quad A A C$ A $A$ A $A C$ A A A A A AC A A A $A \quad A C \quad A \quad A \quad A \quad A A \quad A C \quad A \quad A \quad A \quad A \quad A C$ A $A$ A $A$

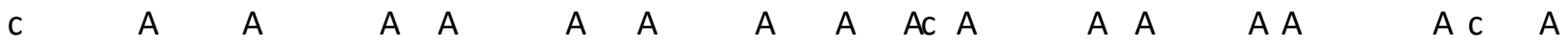
$\begin{array}{lllllllllllll}A & A & A & A & A A & A & C & A & A & A & A & A & A\end{array}$ A A A A C A A A A A A A A A A A A A A $A$ A A AA

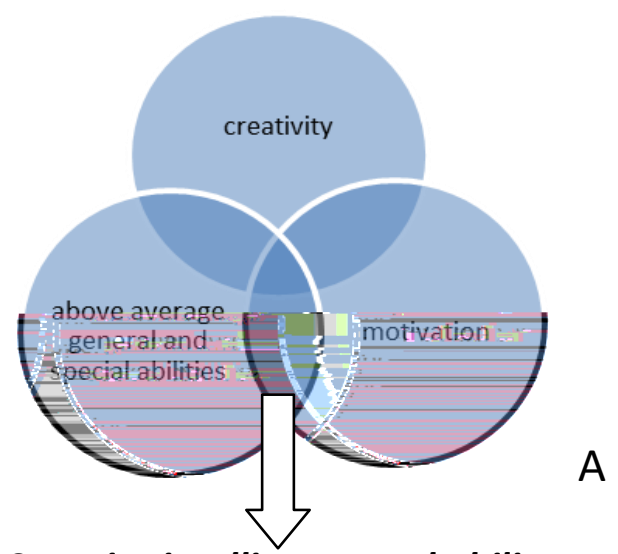

Superior intelligence and ability

G $A \quad A \quad A \quad A \quad A$ 
$\begin{array}{lllllllllllllll}A & A & A & A & A & A & A & A & A A & A & A & A C & A & A & A\end{array}$ A A A A A A A A A A A A A A A A A A A A A A A A A A A A A A

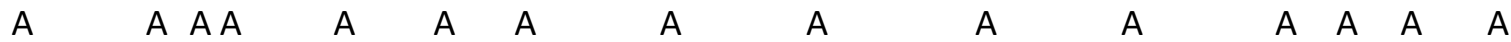

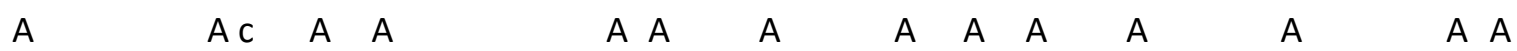

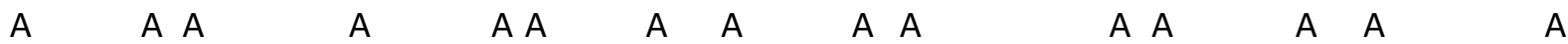
$\begin{array}{lllllllllll}A & A & A & A & A & A & A & A & A & A & A\end{array}$

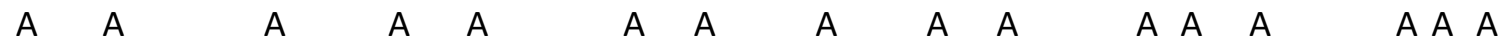

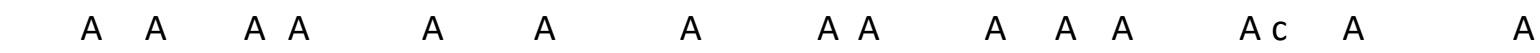
$\begin{array}{lllllllll}A & A & A & A & A & A & A & A & A\end{array}$

A

2. Method
A $A$
A $A \quad A A$
$\begin{array}{llllllll}\text { A } & \text { A } & \text { A } & \text { A } & & & \\ \text { A } & \text { A } & \text { A } & & \text { A A } & \text { A } & \text { A }\end{array}$
A

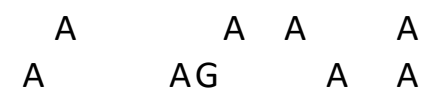

A $\quad A$

A

2.1. Study Group

$\begin{array}{rrrrrrrrrrrrrrrrrrrrrr}A & A & A & A & & A & & A & A & A & A & A & A & A & A & A & A & A \\ A & A & A & A & & A & A & A & A & A & A & A & A & A & A & A & A\end{array}$ $\begin{array}{lllllllll}A & A & A & A & A & A & A & A & A\end{array}$

A

2.2. Data Collection and Analysis

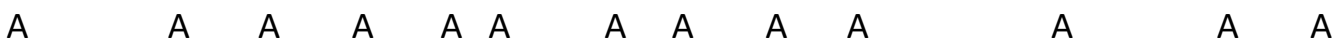

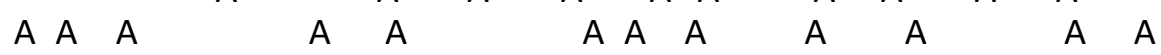

A A A A A A A

C A A A A A A A A A A A

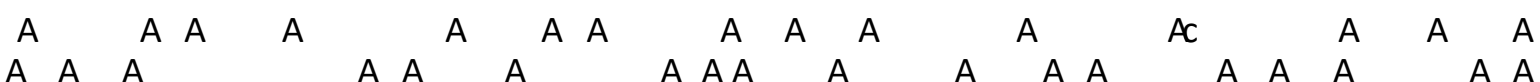
AAA A A A A

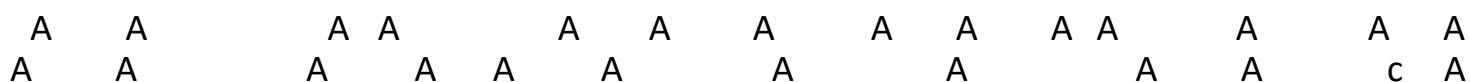

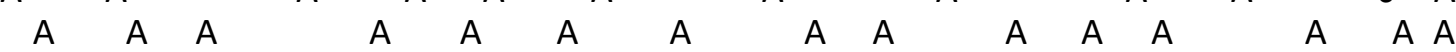
$\begin{array}{lllllllllllllllllllllllll} & A & A & A & A & A G & A & A & A & A & A & A & A & A & A\end{array}$

A A A A A A A A C A A A $A$ AC A A A $A$ A $A$ $\begin{array}{lllllll}A & A & A & A & A & A & A\end{array}$

A

\section{Results}

$\begin{array}{lllllllllllllllll}A & A & A & A & A & A & A & A & A & A & A & A & A & A & A & A\end{array}$ $A A \quad A C$ A A A A A A A A AC A A AC A A

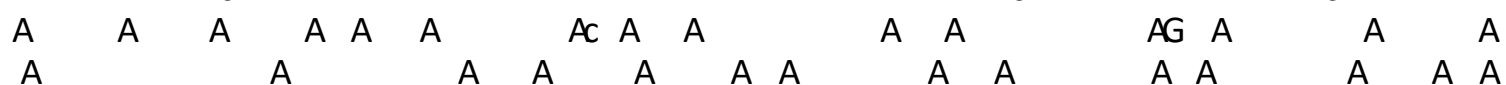
A

A

A 


\begin{tabular}{cccccccccc} 
& & C A A & A A A & A A & A & A & A A A A \\
\hline & A & & & & & A & & A A \\
\hline AAC & AA & A & A & A & & & A & \\
A A & A & & A & & A & & & A & \\
AR AA & & A A & A & A & & A & A \\
\hline
\end{tabular}
$\begin{array}{cccccccccccccccccccc}A & A & A & A & A & C & A A A A & A & A & A & A C & A & A & A & A & A & A & & A \\ A & A & A & A & A & A & A & A & A & A & & A & A & A\end{array}$ A A A A A A A A A A A A A A A A A A

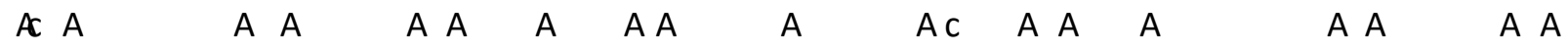
$\begin{array}{llllllllllll}A & A & A & A & A & A & A & A C & A & A & A & A\end{array}$

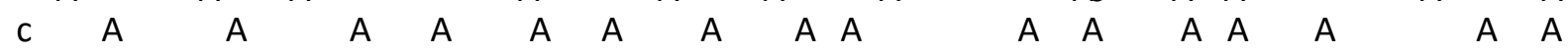

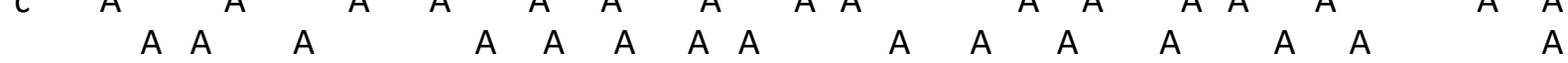
C A A $A$ A A A C A A $\quad A A A A$ A A $A$ A A A A

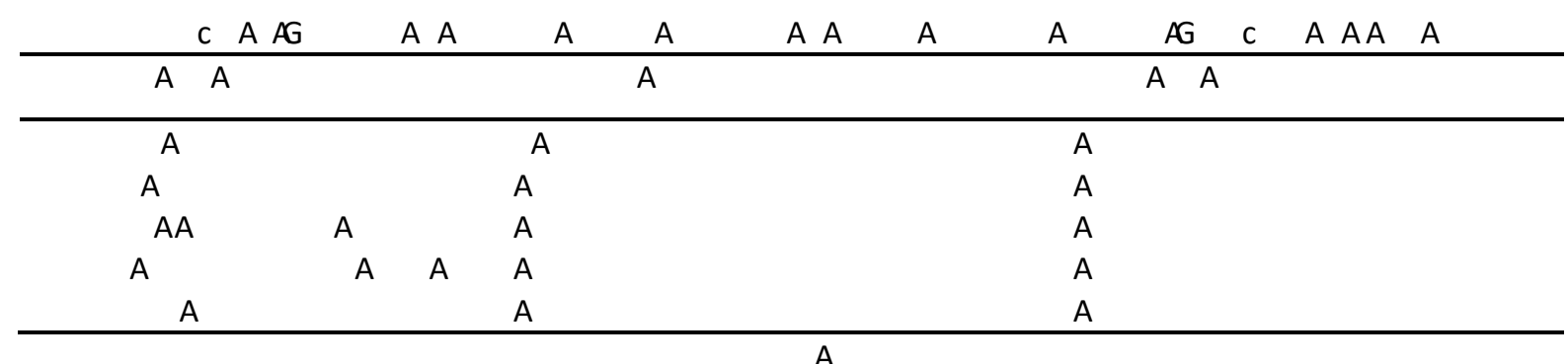

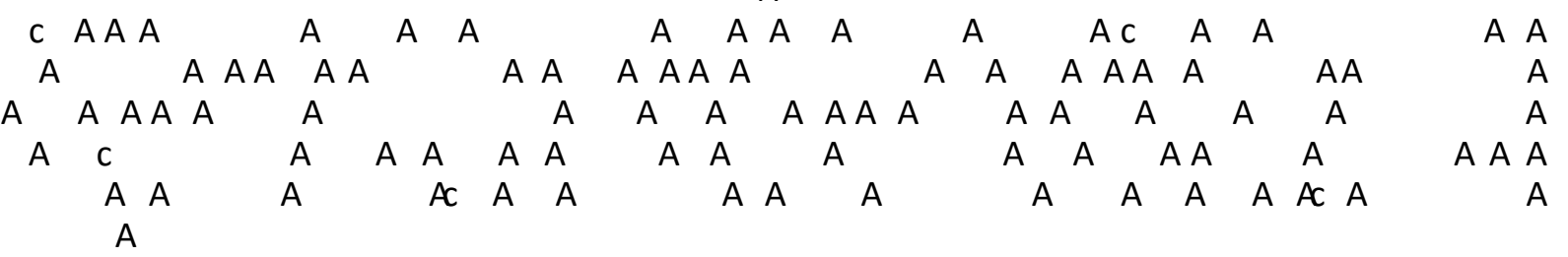

A

\section{Discussion and Conclusion}

$$
\begin{aligned}
& \begin{array}{llllllllllllllllll}
A & A & A & A & & A C & & A & A & A & A & A C & A & A & A & A & A \\
A & A & & A & A & A & A & A & A & A & A & & A & A & A & A & A
\end{array} \\
& \begin{array}{lllllllllll}
A & A & A & A & A & A & A & A & A & A & A
\end{array}
\end{aligned}
$$

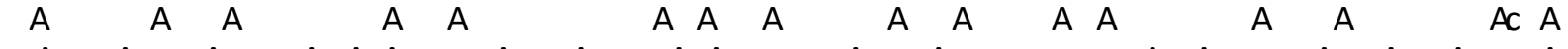

$$
\begin{aligned}
& \begin{array}{llllllllllllllll}
A & A & \subset & A & A & A & A & A & A & A & A & A & A
\end{array} \\
& \text { C A A A A A A A A A A A A A A A A A A } \\
& \text { A } \quad A A \quad A \\
& \begin{array}{llllllllllll}
A & A & A & A & A & A & A C & A & A & A & A & A
\end{array} \\
& \begin{array}{llllllllllllllll}
A & A & A & A & A & A & A & A & A & A & A & A & A & A & A & A
\end{array} \\
& \begin{array}{lllllllllllllllll}
A & C & A & A & A & A & A & A & A & A & A & A & A & A c & A
\end{array} \\
& A A \quad A \quad A \quad A \quad A A \quad A \quad A
\end{aligned}
$$

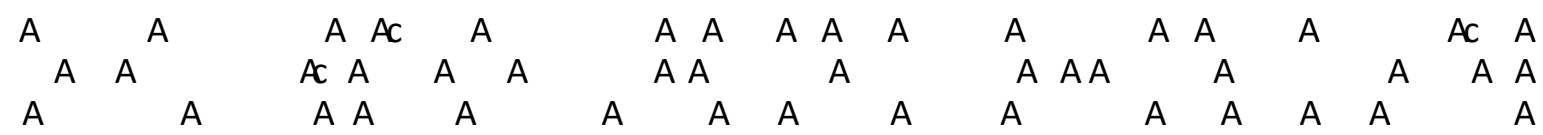


A A A A A A A A

A A

A A A A AA

$A \quad A \quad A$

A $A$

A $\quad A$

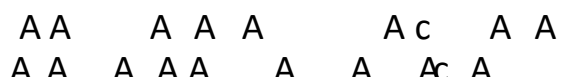

A A A A A

A $A$ A A

A

$\begin{array}{rrrrrrr}A & A & A A & A & A & A C & A \\ A & & A & A & & A & A\end{array}$ $A \quad A \quad A$

A $A$ A

A A A A $\begin{array}{llllllll}A & A & A & A & A & A C & A & A\end{array}$

$\begin{array}{llllllll}A & A & A & A & A & A & A & A\end{array}$

$\begin{array}{lllllll}A & A & A & A & A & A & A\end{array}$

$\begin{array}{llllllllll} & & A & & A & A & & & & A\end{array}$

A

\section{Recommendations}

$$
\begin{aligned}
& \begin{array}{llllllllllllllll}
A & A & A & A & A & A & A & A C & A & A & A & A & A & A & A & A
\end{array} \\
& \begin{array}{llllllllllllllllllll}
A & A & A & A & A & A & A & A & A & A & A & A & A & A
\end{array} \\
& \begin{array}{lllllllllllllllllllllll}
A & A & A & A & A & A & A & A & A & A & A & A & A & A & A & A
\end{array} \\
& \text { A } A \text { A } A \text { A } A \text { A A A A Ac A A A A A A A }
\end{aligned}
$$

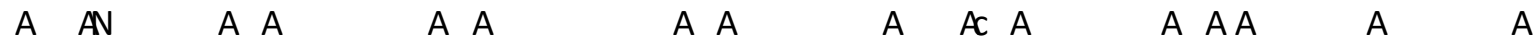

$$
\begin{aligned}
& \text { A } A \text { C } \quad \text { A } \\
& \begin{array}{lllllllllllll}
A & A & A & A & A & A & A & A & A & A & A & A & A
\end{array}
\end{aligned}
$$

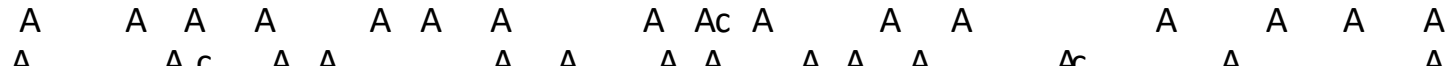

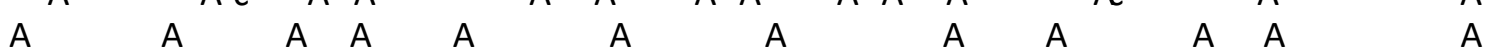

$$
\begin{aligned}
& \begin{array}{lllllllllllllllllllllllll}
A & A & A & A & A & A & A & A & A & A & A & A & A & A & A
\end{array}
\end{aligned}
$$

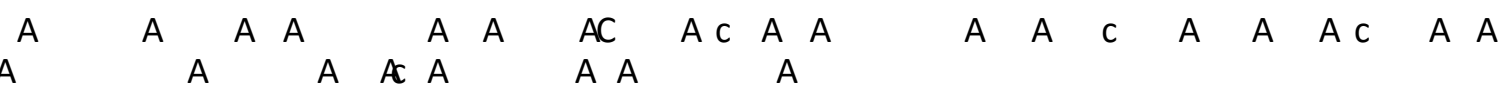

A

\section{References}

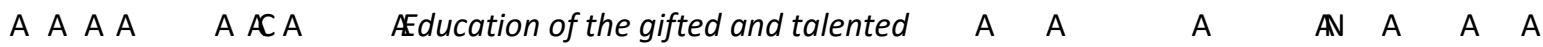
G A A A A A A A AHow to design and evaluate research in education: 6 Ed. A A A

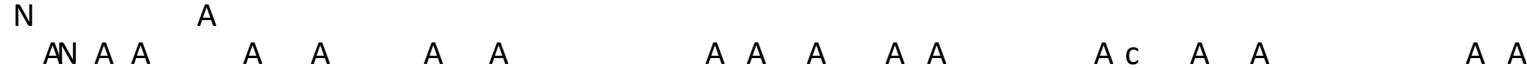
A A A A A A A A A A Anis University Faculty of Education Journal A A $A$

$\begin{array}{lllll}\text { A A A A A } & \text { A } & \text { AG } & & \text { A A } \\ \text { Mathematics, Science \& Technology Education } 12 & \text { A } & \text { A }\end{array}$ A A A A AN A AThe schoolwide enrichment model: A comprehensive plan for educational

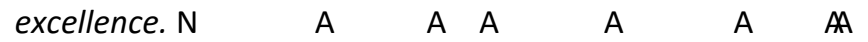
A A AA A A A A A A A A A A A A A Journal for the Education of the Gifted, 23 A A 
$\begin{array}{lllllllllllllll}\text { A A A } & \text { AGA } & \text { A } & \text { A } & \text { A } & \text { A } & \text { A } & \text { A }\end{array}$

A

$A A A \quad A \quad A \quad A \quad A$ C $A A A A$

A A A $A$

\section{A A A}

A

A
A $A$

A
A A AConceptions of Giftedness
A A

A A $A$ A

A $A$

A A AExceptional Children AB A A A
A A

$A$

A

A

$A$

A A 